\title{
Twenty-eight divergent polysaccharide loci specifying within- and amongst-strain capsule diversity in three strains of Bacteroides fragilis
}

\section{Correspondence \\ Sheila Patrick \\ s.patrick@qub.ac.uk}

Received 24 June 2010

Revised 19 August 2010

Accepted 2 September 2010
Sheila Patrick, ${ }^{1}$ Garry W. Blakely, ${ }^{2}$ Simon Houston, ${ }^{1} \dagger$ Jane Moore, ${ }^{1} \ddagger$ Valerie R. Abratt, ${ }^{3}$ Marcelo Bertalan, ${ }^{4} \S$ Ana M. Cerdeño-Tárraga, ${ }^{4} \|$ Michael A. Quail, ${ }_{4}^{4}$ Nicola Corton, ${ }^{4}$ Craig Corton, ${ }^{4}$ Alexandra Bignell, ${ }^{4}$ Andrew Barron, ${ }^{4}$ Louise Clark, ${ }^{4}$ Stephen D. Bentley ${ }^{4}$ and Julian Parkhill ${ }^{4}$

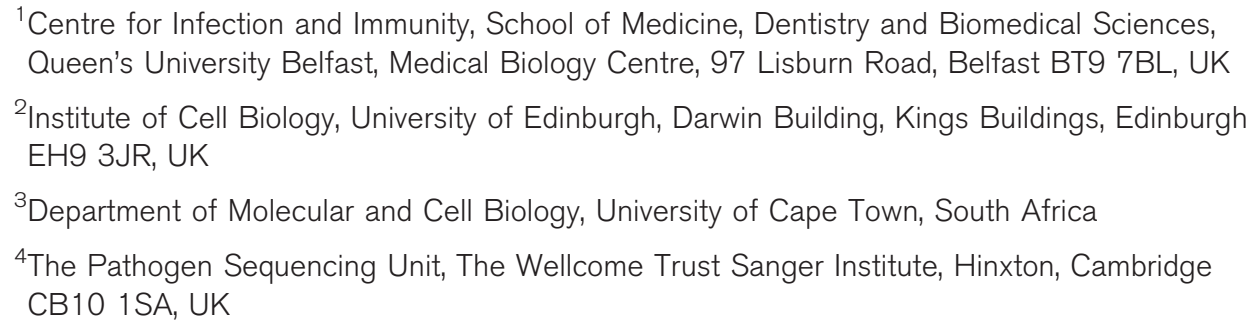
Queen's University Belfast, Medical Biology Centre, 97 Lisburn Road, Belfast BT9 7BL, UK

${ }^{2}$ Institute of Cell Biology, University of Edinburgh, Darwin Building, Kings Buildings, Edinburgh EH9 3JR, UK

${ }^{3}$ Department of Molecular and Cell Biology, University of Cape Town, South Africa

${ }^{4}$ The Pathogen Sequencing Unit, The Wellcome Trust Sanger Institute, Hinxton, Cambridge CB10 1SA, UK

Comparison of the complete genome sequence of Bacteroides fragilis $638 \mathrm{R}$, originally isolated in the USA, was made with two previously sequenced strains isolated in the UK (NCTC 9343) and Japan (YCH46). The presence of 10 loci containing genes associated with polysaccharide (PS) biosynthesis, each including a putative Wzx flippase and Wzy polymerase, was confirmed in all three strains, despite a lack of cross-reactivity between NCTC 9343 and 638R surface PSspecific antibodies by immunolabelling and microscopy. Genomic comparisons revealed an exceptional level of PS biosynthesis locus diversity. Of the 10 divergent PS-associated loci apparent in each strain, none is similar between NCTC 9343 and 638R. YCH46 shares one locus with NCTC 9343, confirmed by mAb labelling, and a second different locus with 638R, making a total of 28 divergent PS biosynthesis loci amongst the three strains. The lack of expression of the phase-variable large capsule (LC) in strain 638R, observed in NCTC 9343, is likely to be due to a point mutation that generates a stop codon within a putative initiating glycosyltransferase, necessary for the expression of the LC in NCTC 9343. Other major sequence differences were observed to arise from different numbers and variety of inserted extra-chromosomal elements, in particular prophages. Extensive horizontal gene transfer has occurred within these strains, despite the presence of a significant number of divergent DNA restriction and modification systems that act to prevent acquisition of foreign DNA. The level of amongst-strain diversity in PS biosynthesis loci is unprecedented.

†Present address: Department of Biochemistry and Microbiology, University of Victoria, Victoria, BC V8W 3P6, Canada.

‡Present address: Department of Molecular Microbiology, John Innes Centre, Norwich Research Park, Colney, Norwich NR4 7UH, UK.

§Present address: Centre for Biological Sequence Analysis, Department of Systems Biology, Technical University of Denmark, Kemitorvet, Building 208, DK-2800 Lyngby, Denmark.

||Present address: European Bioinformatics Institute, Hinxton, Cambridge CB10 1SD, UK.

Abbreviations: ACT, Artemis Comparison Tool; Gl, gastrointestinal; HMMPS, high-molecular-mass polysaccharides; LC, large capsule; MC, micro-capsule; PS, polysaccharide; R-M systems, restriction and modification systems; SC, small capsule; SNP, single nucleotide polymorphism.

The GenBank/EMBL/DDBJ accession number for the sequence and annotation of the Bacteroides fragilis genome is F0312004, and further details are available from http://www.sanger.ac.uk/Projects/B_fragilis/. 


\section{INTRODUCTION}

The obligately anaerobic Gram-negative bacterium Bacteroides fragilis is an important member of the open-ended culture system of the normal resident human gastrointestinal (GI) faecal microbiota, in which the genera Bacteroides and related Parabacteroides predominate both by culture (Patrick et al., 2009) and metagenomic sequencing (Qin et al., 2010). Since the late 1800s, B. fragilis (previously Bacillus fragilis) has also been recognized as an important opportunistic pathogen. It is the major obligately anaerobic Gram-negative bacterium isolated from abscess and soft-tissue infections that arise from contamination by the GI microbiota of normally uncolonized body sites; $B$. fragilis is estimated to account for only between 4 and $13 \%$ of the normal human faecal Bacteroides microbiota by culture and is less abundant than other members of the Bacteroidetes, as demonstrated by metagenomic analysis, but is present in $63-80 \%$ of Bacteroides infections. These include peritonitis subsequent to rupture of an inflamed appendix, serious gynaecological sepsis and brain abscesses (Patrick, 2002; Patrick \& Duerden, 2006). B. fragilis is also the most common cause of anaerobic bacteraemia, with a potential mortality of up to $\sim 30 \%$ (Cheng et al., 2009). Prior to the antibiotic era, such infections severely limited the success of GI and gynaecological surgery due to the high rates of patient death (Patrick \& Duerden, 2006). Metronidazole is generally the most effective antibiotic for both treatment and prophylaxis; a recent worrying development, however, is the report of potentially lethal multi-drug-resistant strains (Katsandri et al., 2006; Wareham et al., 2005). In contrast to $B$. fragilis, other Bacteroidetes more abundant in the GI tract, such as Bacteroides vulgatus and Parabacteroides distasonis (formerly Bacteroides distasonis; Sakamoto \& Benno, 2006), are rarely isolated from infection (Patrick \& Duerden, 2006). Characteristics of $B$. fragilis that may play a role in its success as an opportunistic pathogen include the release of degradative enzymes such as fibrinogenolysin (Houston et al., 2010), enterotoxin production, evasion of complement-mediated killing and phagocytosis, the induction of abscess formation, and extensive within-strain variation of surface proteins and polysaccharides (PSs) (Wexler, 2007). Multiple invertible promoters play a key role in generating the observed antigenic variation (Cerdeño-Tárraga et al., 2005; Patrick et al., 2003). The marginal electron-dense layer, or micro-capsule (MC), of approximately $35 \mathrm{~nm}$ in size, outwith the outer membrane and not visible by light microscopy (Patrick et al., 1986; Lutton et al., 1991), is linked to complement resistance, and at least one of these antigenically variable PSs is associated with abscess formation (Wexler, 2007). In addition to MC, antigenically distinct and within strain-variable large capsules (LCs) and small capsules (SCs) are visible by light microscopy when cultures are grown in a glucose-defined medium (Patrick et al., 1986). The LC is associated with resistance to phagocytic uptake and killing (Reid \& Patrick, 1984). A putative PS chain length-determining Wzz protein homologue is involved in the production of high-molecular-mass polysaccharides (HMMPS) associated with the MC; a $w z z$ deletion mutant in NCTC 9343 does not produce HMMPS, but still produces SC and LC (Patrick et al., 2009). Phase variation of LC production is also under invertible promoter control (Patrick et al., 2009).

We now present the complete genome sequence of a $B$. fragilis plasmid-free spontaneous rifampicin-resistant mutant, 638R (Privitera et al., 1979; also known as TM400; Tally et al., 1982), which was generated at the Institut Pasteur (Paris, France) from a clinical isolate originating from Chicago, USA (Stiffler et al., 1974). Comparison of $638 \mathrm{R}$ with two published $B$. fragilis genome sequences, NCTC 9343 (Cerdeño-Tárraga et al., 2005), isolated from peritoneal infection in London (UK), and a blood culture isolate, YCH46, isolated in Yamaguchi (Japan; Kuwahara et al., 2004), reveals an unprecedented diversity of PSs amongst the three strains.

\section{METHODS}

Bacterial strains and culture conditions. B. fragilis 638R (isolated from an abdominal abscess), kindly gifted by C. J. Smith, East Carolina University, USA, was derived from a culture supplied to him by M. Sebald (Institut Pasteur, Paris, France) in 1983. B. fragilis YCH46, a bacteraemia isolate from Yamaguchi Prefecture (Japan), was kindly gifted by T. Kuwahara, University of Tokushima (Japan). An early freeze-dried stock culture of B. fragilis National Collection of Type Cultures (NCTC) 9343, originally isolated from an abdominal infection at St Bartholomew's Hospital, London, UK, in 1955, was obtained from the NCTC, London, UK.

B. fragilis was cultured in either supplemented brain heart infusion (BHI-S) or glucose defined medium (DM; Van Tassell \& Wilkins, 1978). B. fragilis was cultured in a MACS MG-1000 anaerobic workstation (Don Whitley) at $37{ }^{\circ} \mathrm{C}$ in an atmosphere of $80 \%$ nitrogen, $10 \%$ carbon dioxide and $10 \%$ hydrogen. Clinical isolates $(n=23)$ and GI tract isolates $(n=9)$ were cultured in DM broth, and the proportion of capsulate cells was estimated by five random field counts (Patrick \& Reid, 1983). Enrichment of populations with different sizes of capsules was carried out using discontinuous Percoll density-gradient centrifugation, as previously described (Patrick \& Reid, 1983; Patrick et al., 2009).

B. fragilis $638 \mathrm{R}$ complete genome sequencing. $B$. fragilis $638 \mathrm{R}$ was grown in DM as described above, and DNA was isolated using a modification of the basic protocol for preparation of genomic DNA from bacteria described by Ausubel et al. (1992). In brief, bacterial cells were lysed in $10 \mathrm{mM}$ Tris/HCl/1 mM EDTA buffer ( $\mathrm{pH} 8.0$ ) containing SDS $(0.5 \%)$, lysozyme $\left(4 \mathrm{mg} \mathrm{ml}^{-1}\right)$ and proteinase $\mathrm{K}$ $\left(0.1 \mathrm{mg} \mathrm{ml}^{-1}\right)$. PS was precipitated using cetyltrimethylammonium bromide (CTAB) and DNA was extracted with chloroform: isoamyl alcohol and phenol:choloroform: isoamyl alcohol mixtures. DNA was precipitated using 2-propanol, spooled out with a glass rod and washed in ethanol. The initial genome assembly was obtained from 86560 paired-end sequences (giving ninefold coverage) derived from two pUC18 genomic shotgun libraries (with insert sizes ranging from 1.4 to $3.6 \mathrm{~kb}$ ) using BigDye terminator chemistry on ABI 3700 automated sequencers. A total of 1474 paired-end sequences from a pBACe3.6 library were used as a scaffold. All identified repeats were bridged by read-pairs or end-sequenced PCR products. A further 14870 sequencing reads were generated during finishing. The 
sequences were assembled, finished and annotated as described previously, using Artemis to collate data and facilitate annotation (Cerdeño-Tárraga et al., 2005). The DNA and encoded protein sequences of related species were compared using the Artemis Comparison Tool (ACT; Carver et al., 2005). A percentage identity cut-off was not set for the ACT comparisons. Whole-genome comparisons were performed using BLAST with default parameters, and all matches with a BLAST score $>100$ were collected and displayed. Non-homologous regions showed no matches above this threshold. Orthologous gene sets were calculated by reciprocal best match FASTA comparisons, with subsequent manual curation. Pseudogenes had one or more mutations that would ablate expression; each of the inactivating mutations was subsequently checked against the original sequencing data.

Regions associated with PS biosynthesis were numbered in the primary annotation of the NCTC 9343 genome sequence; these numbers have been retained along with the letters subsequently designated for PS loci A-J (Patrick et al., 2003; Cerdeño-Tárraga et al., $2005)$ to enable cross-reference. Additional loci reported herein have been assigned both letter and number designations.

Microscopy. Bacteria grown in DM broth to late-exponential phase were fixed with glutaraldehyde and osmium tetroxide, and prepared for transmission electron microscopy as previously described (Patrick et al., 1986). Capsule smears were performed on DM-grown cultures using Eosin-carbol fuchsin negative stain. Hybridoma cell lines secreting mouse $\mathrm{mAb}$ were generated by inoculation of whole bacterial cells, as previously described (Lutton et al., 1991) under UK Government Home Office Personal and Project Licences and with local ethical approval. Immunofluorescence labelling was carried out as described by Lutton et al. (1991) with the following modifications. Bacterial cells, grown in $\mathrm{DM}$ broth, were suspended at $\mathrm{OD}_{600} 0.3$ $\left(\sim 5 \times 10^{8}\right.$ cells $\left.\mathrm{ml}^{-1}\right)$ in PBS. Bacterial suspension $(10 \mu \mathrm{l})$ was applied to a Teflon-coated multiwell slide (ICN Biomedicals). The slides were then dried at $37{ }^{\circ} \mathrm{C}$, and cells were fixed by submerging in $100 \%$ methanol at $-20{ }^{\circ} \mathrm{C}$ for $20 \mathrm{~min}$. After air drying, the cells were immunolabelled with $\mathrm{mAb}$ hybridoma supernatants $(30 \mu \mathrm{l}$ of a $1: 10$ dilution in PBS), incubated at $37{ }^{\circ} \mathrm{C}$ in a humidified container for $45 \mathrm{~min}$, and then washed in PBS with agitation for $20 \mathrm{~min}$. Secondary antibody, 1:100 goat anti-mouse IgG-FITC (Sigma), and 4\% (v/v) Evans blue counter stain in PBS $(30 \mu \mathrm{l})$ were added to each well. Slides were incubated at $37{ }^{\circ} \mathrm{C}$ in a humidified container for $45 \mathrm{~min}$ and washed as before, mounted in glycerol-PBS anti-bleaching mounting fluid (Citifluor, Agar Scientific) and examined at $\times 1000$ magnification (Leitz Ortholux fluorescence microscope). Images were captured using a Nikon DMX 1200 digital camera and Lucia G/F software.

PCR amplification of the LC invertible promoter region. Primers were designed which amplified the upstream region of BF2782 with the promoter in both the ON and OFF orientations, as follows: BF2782 'Promoter ON' primers, BF2782 ON-For (5'-AAAAAAGGATCCATTAGGTAATAAATGCGGAATAGCG-3') and BF2782 ON-Rev (5'-AAAAAAGCATGCAACTCTGTGTTCTCTGTGGTG-3'). This primer set amplifies a $1.85 \mathrm{~kb}$ DNA sequence upstream of BF2782 which has the promoter only in the ON position with respect to the BF2782 start codon; BF2782 'Promoter OFF' primers, BF2782 OFFFor (5' -AAAAAAGGATCCCACTTAATAGAAATATTAGTC-3') and BF2782 OFF-Rev (5'-AAAAAAGCATGCAACTCTGTGTTCCTCTGTGGTG-3'). This primer set amplifies a $1.75 \mathrm{~kb}$ DNA sequence upstream of BF2782 which has the promoter only in the OFF position with respect to the BF2782 start codon. DNA extraction and PCR were carried out as detailed in Patrick et al. (2009), with the following modifications. Purified genomic template DNA from each of the enriched capsular populations and strains $638 \mathrm{R}$ and $\mathrm{YCH} 46$ was diluted in autoclaved ultrapure water to give final concentrations of
$100 \mu \mathrm{g} \mathrm{ml}^{-1}$. PCR mixtures were prepared using Platinum Taq High Fidelity DNA polymerase (Invitrogen) according to the manufacturer's instructions. The reaction mixtures were incubated at $94{ }^{\circ} \mathrm{C}$ for $3 \mathrm{~min}$ followed by 35 cycles of PCR amplification at $94{ }^{\circ} \mathrm{C}$ for $30 \mathrm{~s}$, annealing at $60{ }^{\circ} \mathrm{C}$ for $15 \mathrm{~s}$, extension at $68{ }^{\circ} \mathrm{C}$ for $1 \mathrm{~min}$, and a final extension step at $68{ }^{\circ} \mathrm{C}$ for $10 \mathrm{~min}$. PCR products were detected as described in Patrick et al. (2009).

Transformation of strain 638R with pVA2198-BF2782. Transformation was performed using pVA2198-BF2782, with the bacteria maintained in an anaerobic atmosphere throughout, as described in Patrick et al. (2009), with the following modifications. Under anaerobic conditions, B. fragilis, Ocr protein $(8-16 \mu \mathrm{g})$, and plasmid (3.25 $\mu \mathrm{g}$ of pVA2198-BF2782 per electroporation) were added to the electroporation cuvette and mixed. As positive controls, $B$. fragilis $638 \mathrm{R}$ and NCTC 9343 were also electroporated with pVA2198 (500 ng plasmid and 8-16 $\mu$ g Ocr protein). Transformed cells were initially plated onto BHI-S agar plates containing $10 \mu \mathrm{g}$ erythromycin $\mathrm{ml}^{-1}$ with or without $20 \mu \mathrm{g}$ rifampicin $\mathrm{ml}^{-1}$. Transformants were subcultured by patch plating individual colonies onto glucose DM agar plates containing $10 \mu \mathrm{g}$ erythromycin $\mathrm{ml}^{-1}$ with or without $20 \mu \mathrm{g}$ rifampicin $\mathrm{ml}^{-1}$ prior to performing capsule smears and microscopy.

\section{RESULTS AND DISCUSSION}

\section{Within-strain antigenically variable MC comparisons}

Within-strain antigenic variation of MC expression, driven by invertible promoters, is evident in B. fragilis NCTC 9343 and a range of other clinical isolates by reaction with a suite of mAbs. The rate of DNA inversion is sufficiently rapid to generate variants during growth of a single colony on glucose DM agar; thus, within a single population there are both promoter ON and OFF bacterial cells for multiple loci. As a result, immunofluorescence microscopy reveals, within a single population, labelling of variable numbers of bacterial cells with multiple different mAbs (Patrick et al., 1999). B. fragilis 638R, however, does not react with the suite of mAbs that react with the MC of NCTC 9343 and also with a range of isolates from Northern Ireland (Patrick et al., 1995); nevertheless, an electron-dense MC outwith the outer membrane is clearly evident in 638R by electron microscopy (Fig. 1). As previously described for NCTC 9343 (Patrick et al., 2003; Cerdeño-Tárraga et al., 2005), eight separate MC biosynthesis loci were also identified in the $638 \mathrm{R}$ genome, each characterized by the presence of putative $\mathrm{O}$-antigen flippase genes $(w z x)$ and polysaccharide polymerases $(w z y)$, indicating the production of PSs, exported by Wzy-dependent methods (Whitfield, 2006). Whole-genome comparison, using ACT, revealed conservation of the upstream promoter regions, including the invertible regions associated with seven of the loci (Patrick et al., 2003). As in NCTC 9343, there is evidence of active DNA inversion in the genome of the $638 \mathrm{R}$ population from which the DNA used for genome sequencing was extracted. Invertible promoters in both the ON and OFF positions are present in the $638 \mathrm{R}$ shotgun sequence data. In addition, the invertase finA (Patrick et al., 2003) 638R 2788, equivalent 

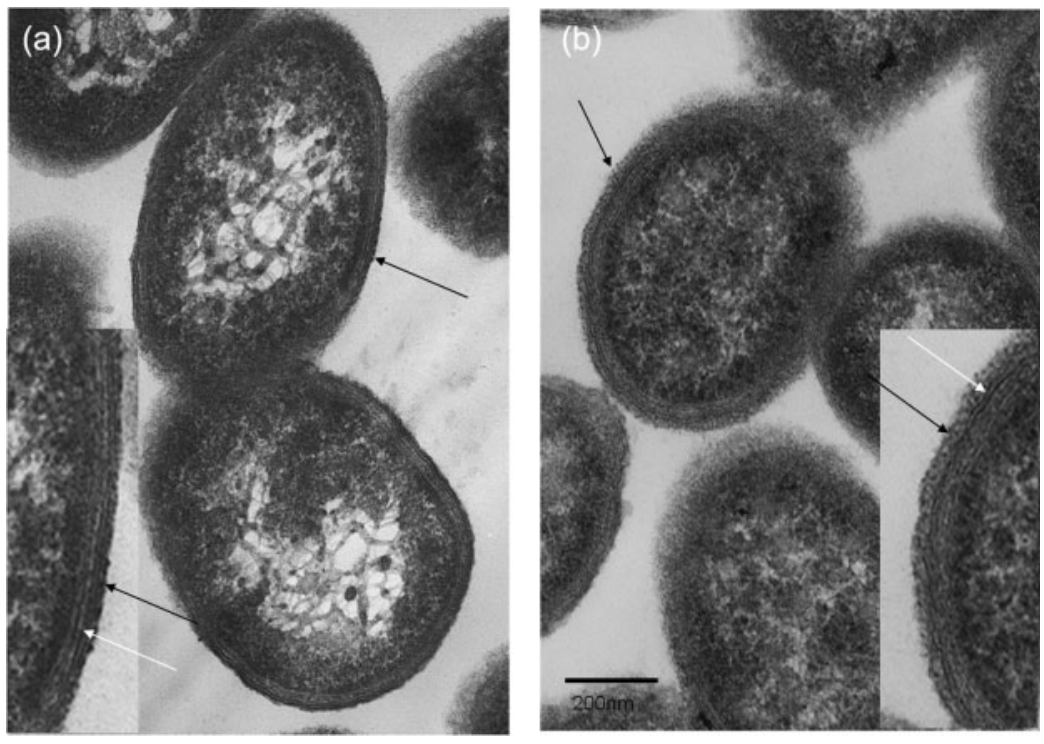

\begin{abstract}
Fig. 1. Electron micrographs of ultrathin sections of $B$. fragilis. (a) NCTC 9343 MCenriched population; (b) $B$. fragilis 638R. Note the presence of an electron-dense layer (black arrow) outwith the outer membrane (white arrow) in both strains. Bar, $200 \mathrm{~nm}$.
\end{abstract}

to NCTC 9343 BF2779, which drives the DNA inversion at these loci, is conserved. The putative transcriptional regulators $u p(a-h) Z$ and $u p(a-h) Y$, located at the start of seven of the PS biosynthesis loci, are also conserved, with the exception of the C-terminal region of the $638 \mathrm{R}$ $u p b Z$. There is, however, a striking lack of DNA sequence identity between strain 638R and strain NCTC 9343 in the remainder of each of the loci as revealed by ACT analyses (Fig. 2a-h, Table 1). Within the non-identical regions, genes putatively involved in PS formation are present, potentially representing a different set of eight PSs in strain 638R. This divergence is likely to explain the lack of crossreactivity of 638R with the NCTC 9343 antibodies. The PSassociated region PSJ/10, which encodes the only Wzz homologue, and which is involved in the generation of HMMPS associated with the MC, but not SC or LC formation (Patrick et al., 2009), is however conserved amongst all three strains (results not shown).

Seven mAbs, generated using whole cells of $638 \mathrm{R}$, reacted with HMMPS from 638R as demonstrated by proteinase $\mathrm{K}$ digestion, PAGE and immunoblotting (results not shown). Immunofluorescence microscopy revealed within-population antigenic variation in 638R, but not cross-reaction with NCTC 9343 (Fig. 3). Further genomic comparison with the previously sequenced Japanese strain, YCH46, which also contains eight $\mathrm{MC}$ loci, revealed only two shared loci amongst the three strains; 638R PSC/8 is shared with YCH46, and the NCTC 9343 PSB/4 locus is shared with $\mathrm{YCH} 46$. The existence of conserved loci between NCTC 9343 and YCH46, as observed using ACT, was confirmed experimentally by immunofluorescence microscopy using a mAb that reacts with NCTC 9343 PSB/4 (Fig. 4). The zwitterionic amino sugar-containing NCTC 9343 $\mathrm{PSA} / 2$, which is involved in abscess formation (Coyne et al., 2001) and which also protects animals from experimental colitis (Mazmanian et al., 2008), is not conserved amongst the three sequenced strains and is present in NCTC 9343 alone (Fig. 2a). Kuwahara et al. (2004) suggested that the presence of putative aminotransferase and dehydrogenase genes in five of the YCH46 PS loci could potentially generate five different abscess-inducing PSs. On this basis, four of the $638 \mathrm{R}$ loci that contain putative aminotransferases and dehydrogenases may produce a different set of abscess-inducing PSs.

\section{Within-strain phase-variable LC and SC comparisons}

As with antigenic variation of the $\mathrm{MC}$, phase variation of the LC and SC in NCTC 9343 occurs during growth of a single population in which both phase $\mathrm{ON}$ and phase OFF bacterial cells are evident. Neither 638R nor YCH46 produces an LC when cultured in a glucose DM (Van Tassell \& Wilkins, 1978); nor is it possible to enrich for an LC population using Percoll density-gradient centrifugation (Patrick \& Reid, 1983; Fig. 5). Three key genes are implicated in LC production by B. fragilis NCTC 9343: BF2782, a putative initiating glycosyltransferase WbaP homologue; BF2783, a putative Wza polysaccharide export/ assembly protein homologue; and BF2784, a putative polysaccharide co-polymerase Wzc homologue.

In NCTC 9343, expression of the LC is under the control of an invertible promoter, driven by Tsr19 (BF2780), a tyrosine site-specific recombinase (Chatzidaki-Livanis et al., 2008). ACT comparisons indicated that the invertible promoter and the recombinase are conserved in 638R and YCH46. Comparison with the published YCH46 sequence, however, revealed that duplication of the inverted repeats present at one end of the NCTC 9343 invertible region is apparent at both ends of the invertible promoter region in YCH46. How this affects inversion of this region is not known. The invertible promoter region which mediates this within-strain variable expression of the LC is in the 'OFF' orientation in all three genome sequences. This is to 
be expected in the NCTC 9343 genome, as the DNA used for the sequencing project was purified from a population in which fewer than $1 \%$ of the cells had the LC phenotype (Cerdeño-Tárraga et al., 2005); therefore, it is unlikely that inversion would be evident in the shotgun sequencing. To determine whether active inversion occurred within the populations, PCR amplification of purified genomic DNA from populations of the three strains, using primer pairs designed to amplify the BF2782-equivalent gene with its promoter in both the ON and OFF orientations, resulted in the detection of amplicons of the predicted size for both orientations (Fig. 6). The promoter is therefore present in both orientations in populations of all three strains. This indicates the potential for phase-variable expression of the downstream genes, the first of which is the putative WbaP initiating glycosyltransferase. Wza proteins form major multimeric ring-like structures in the outer membrane that are thought to function as export channels in translocation of HMMPS across the outer membrane, and possibly also in assembly of the capsule on the cell surface (Drummelsmith \& Whitfield, 2000); a BF2782 deletion mutant generated in NCTC 9343 does not exhibit the LC phenotype (Patrick et al., 2009). The equivalent gene in 638R, BF638R 2790, contains a single nucleotide polymorphism (SNP) that results in a stop codon, located within the $5^{\prime}$ region of a putative $\mathrm{C}$-terminal sugar transferase domain, potentially resulting in the truncation of approximately $75 \%$ of this functionally important domain. This stop mutation may therefore abolish the enzymic function of the glycosyltransferase, which may in turn account for the lack of LC production by $638 \mathrm{R}$.

Complementation of an NCTC 9343-BF2782 deletion mutant with pVA2198-BF2782, containing the invertible promoter in the $\mathrm{ON}$ position, restored the phase-variable LC phenotype (Patrick et al., 2009); however, transformation of 638R with this plasmid did not result in an LC phenotype. It is possible that the stop codon in BF638R 2790 has polar effects on transcription of the additional downstream genes, although this will require confirmation by analysis of transcripts.

The reason for the lack of production of the LC in $\mathrm{YCH} 46$ is not immediately evident from the genome sequence, as the BF2782-equivalent gene (BF YCH46 2767) has an identical sequence to that of NCTC 9343. There are, however, SNPs in the downstream putative Wza BF YCH46 2768 (BF2783 equivalent), and in BF 638R 2793, equivalent to BF2784, the putative Wzc polysaccharide co-polymerase. These SNPs effect amino acid substitutions. Whether or not these alter the structure and biological function of the key capsular biosynthesis proteins remains to be determined. It may be that the lack of LC phenotype and presence of SNPs reflect the degree of laboratory subculture of these strains away from the selective pressures of the human host. All recent clinical and GI tract isolates of $B$. fragilis $(n=32)$ examined to date produced the LC in proportions varying from 30 to $1 \%$ or less (results not shown).
Populations of NCTC 9343 expressing an SC can be obtained at the $20-40 \%$ interface layer of a Percoll discontinuous density gradient after centrifugation (Patrick et al., 1986). Strains 638R and YCH46 also had an observable SC by light microscopy after negative staining, when sampled from the $20-40 \%$ interface, although the SC was more irregular in strain 638R (Fig. 5). Labelling of the 638R and YCH46 SC populations with a mAb specific for the SC of NCTC 9343 (Reid et al., 1987), however, revealed that these populations were not enriched for the NCTC 9343 SC-associated epitope (Fig. 5). As previously noted for NCTC 9343 (Patrick et al., 1986), the SC population of strain $638 \mathrm{R}$ could be enriched by subculture from the $20-40 \%$ Percoll interface layer into glucose DM; however, YCH46 failed to subculture. This may relate to the poor growth of YCH46 in glucose DM. YCH46 is growth-limited in this medium when compared with NCTC 9343 and 638R with respect to growth rate (mean doubling time of approximately $4 \mathrm{~h}$ as opposed to 1.6 and $1.2 \mathrm{~h}$, respectively) and total viable count attained in late-exponential phase.

\section{Comparison of two PS loci yet to be associated with capsule type}

The PS loci I/9 and K/11 are two further divergent loci that appear to lack invertible promoters and have yet to be associated with a particular capsular type (Fig. 7, Table 1).

In PS locus I/9 (Fig. 7a), comparison of 638R and YCH46 reveals conservation in a 12-gene region (from BF YCH46 2776 and BF 638R 2800) downstream of a predicted promoter, which is divergent in NCTC 9343. In YCH46, an inserted conjugative transposon disrupts the twelfth gene, BF YCH46 2787, a putative fucosyltransferase. This may inhibit or prevent synthesis of the PSI/9 PS or simply alter the structure. Interestingly, the next gene immediately downstream of BF YCH46 2787 in the locus is a putative recombinase. If the transposon is mobile, insertion and loss could generate variable expression of the putative PS encoded at this locus.

In addition, two acetyltransferase-related proteins within the PSI/9 region, otherwise conserved between $\mathrm{YCH} 46$ and $638 \mathrm{R}$, are disrupted in 638R. A point mutation in BF638R 2810 generates a stop codon. In addition, in the BF638R region equivalent to BFYCH46 2780, a frameshift is generated by an additional cytosine in a run of 4 Cs. The presence or absence of an $O$-acetyl group on a sugar residue is a well-documented mediator of capsular variation in other bacteria. For example, Escherichia coli slipped-strand mispairing/replicative slippage can occur during DNA replication and results from heteropolymeric repeats in the region upstream of an $O$-acetyltransferase; this generates within-strain antigenic variation of the K1 PS (Vimr \& Steenbergen, 2006). Homopolymeric repeat regions, within and outwith coding sequences, are also linked to variation generated by slipped-strand misparing/ replicative slippage in other bacteria (van der Woude \& 
(a)

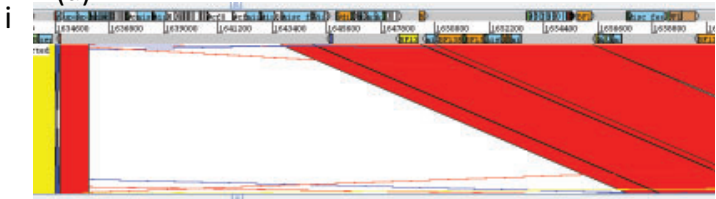

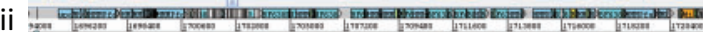

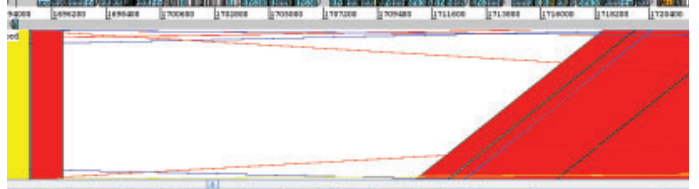

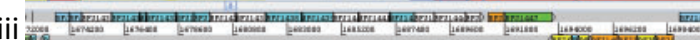

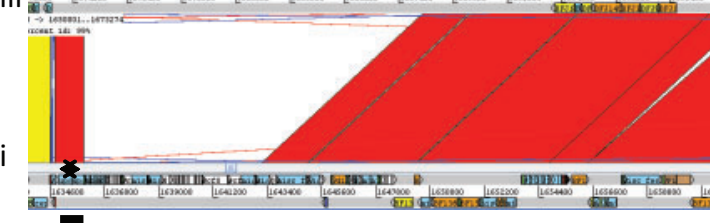

(c)

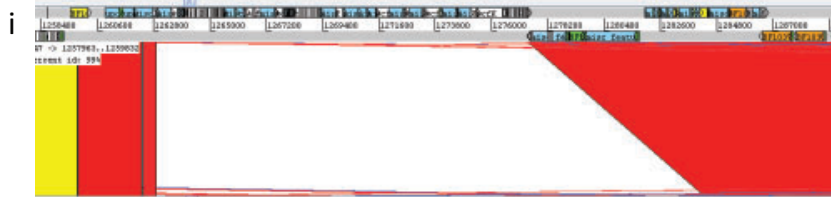

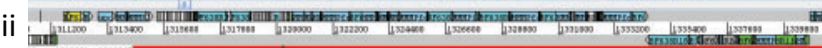

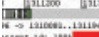
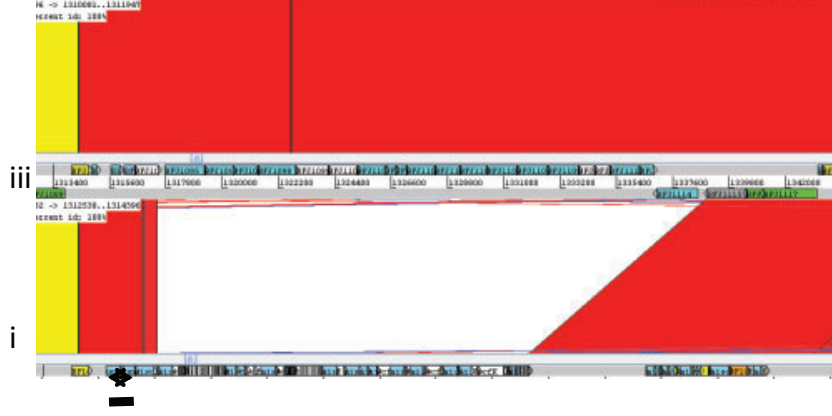

(e)

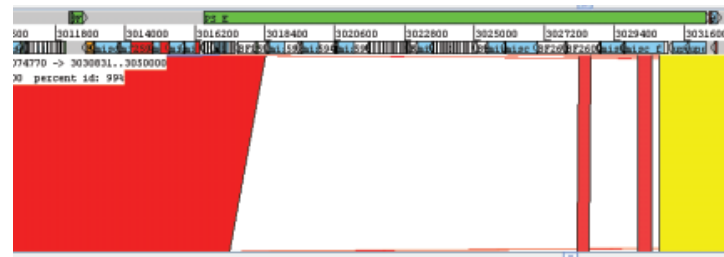

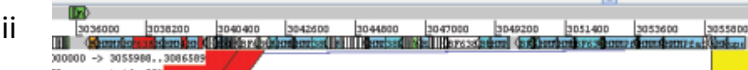

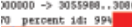
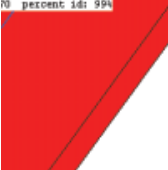

ii

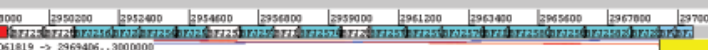

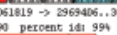
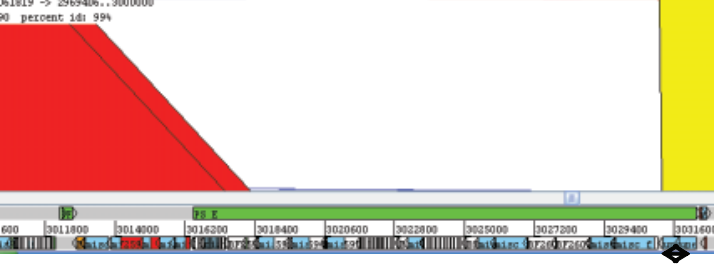

(b)
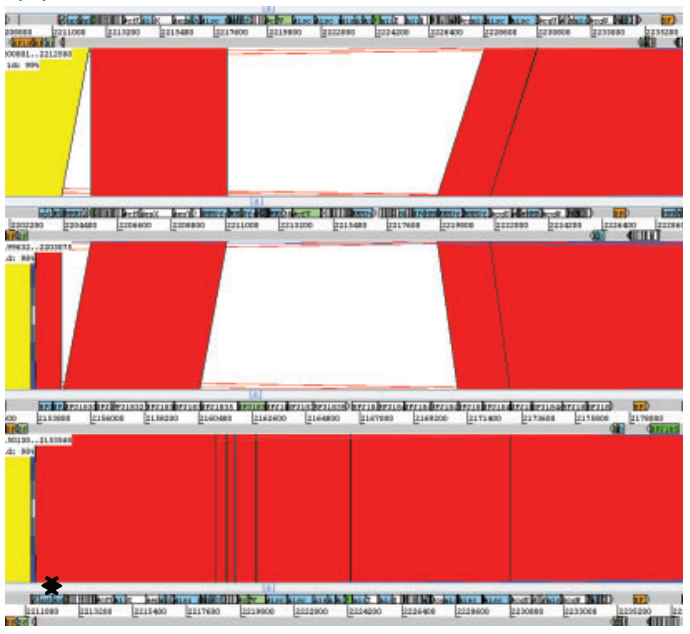

(d)

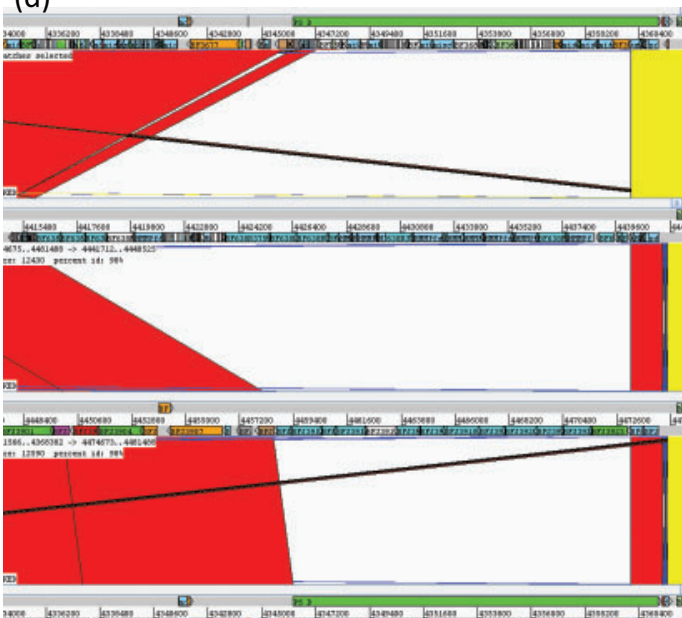

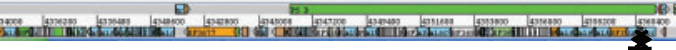

(f)
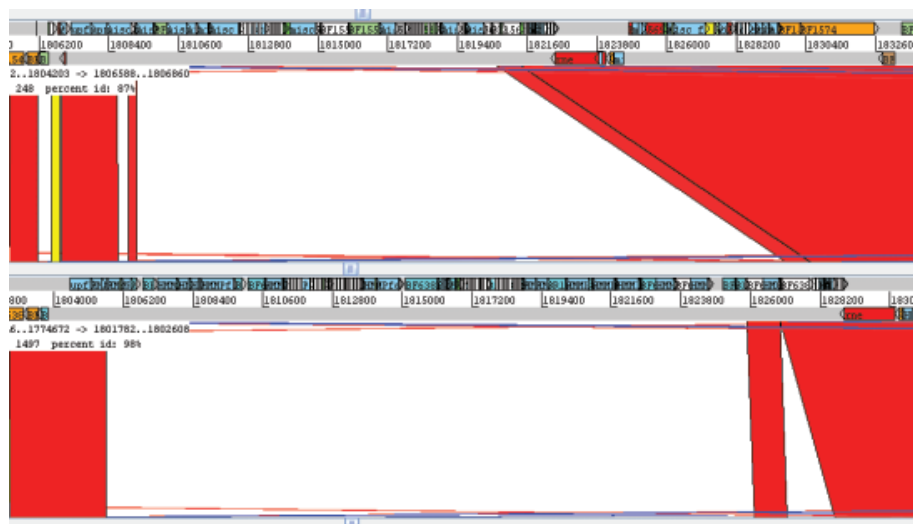

1 Exक L1975460 L177600

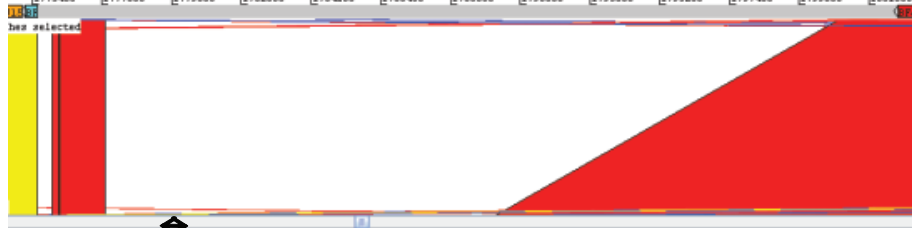

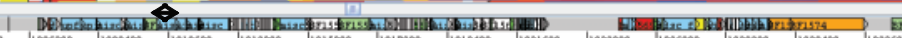

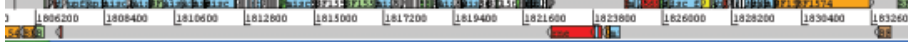

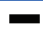


(g)

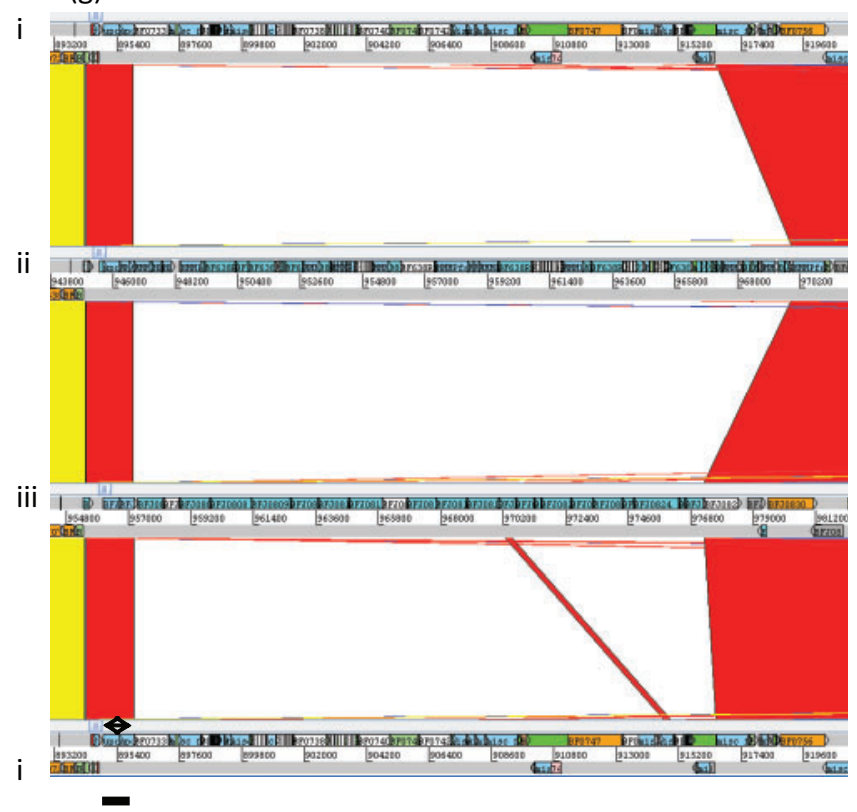

(h)

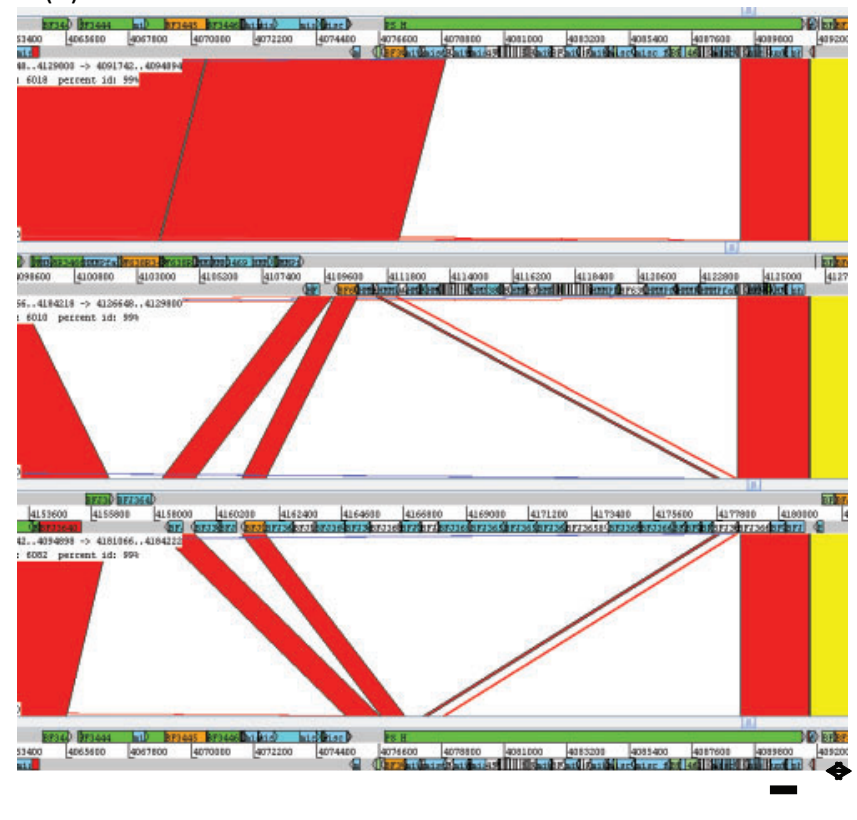

Fig. 2. Four-way ACT genome comparison of within-strain variable $M C$-associated loci. (a) $P S A / 2$, (b) $P S B / 4$, (c) $P S C / 8$, (d) PSD/5, (e) PSE/1, (f) PSF/6, (g) PSG/7, (h) PSH/3 of B. fragilis. (i) NCTC 9343, (ii) 638R, (iii) YCH46. Red or yellow colouration between sequences indicates sequence identity, no colour indicates divergent sequence, dark blue indicates an inverted promoter region. Line, conserved transcriptional regulator region. Note: sequence identity was only evident between strains NCTC 9343 and $\mathrm{YCH} 46$ at locus PSB/4, and strains $638 \mathrm{R}$ and $\mathrm{YCH} 46$ at locus PSC/8.

Baumler, 2004). A mechanism such as this could generate antigenic variation of a PS encoded by this locus in strain YCH46.

In NCTC 9343, the PSI/9 locus, equivalent to the 12 genes of YCH46 and 638R, encodes an entirely different set of 18 genes associated with PS synthesis (from NCTC 9343 BF2791), indicating potential production of a different PS.

All three strains encode a putative Wzx flippase (divergent in NCTC 9343) in this region, and there are genes containing 8-12 transmembrane segments, characteristic of Wzy

Table 1. PS biosynthesis-associated loci in B. fragilis NCTC 9343, 638R and YCH46

\begin{tabular}{|c|c|c|c|c|}
\hline \multirow[t]{2}{*}{ Locus } & \multicolumn{3}{|c|}{$\mathrm{CDSs}^{*}$} & \multirow[t]{2}{*}{ Phenotype association } \\
\hline & NCTC 9343 & $638 R$ & YCH46 & \\
\hline $\mathrm{B} / 4$ & $1895-1914$ & $1864-1883$ & $1830-1848$ & $\mathrm{MC}$ \\
\hline $\mathrm{C} / 8$ & $1011-1026$ & $1076-1095$ & $1094-1113$ & $\mathrm{MC}$ \\
\hline $\mathrm{D} / 5$ & $3682-3697$ & $3750-3773$ & $3911-3923$ & $\mathrm{MC}$ \\
\hline $\mathrm{G} / 7$ & $0733-0752$ & $0778-0810$ & $0805-0827$ & $\mathrm{MC}$ \\
\hline $\mathrm{H} / 3$ & $3451-3464$ & $3472-3487$ & $3646-3665$ & $\mathrm{MC}$ \\
\hline $\mathrm{I} / 9$ & $2791-2817$ & $2800-3826$ & $2776-2935$ & Unknown \\
\hline $\mathrm{J} / 10$ & $1706-1710$ & $1710-1714$ & $1700-1704$ & $\mathrm{MC}$ \\
\hline $\mathrm{K} / 11$ & $2048-2062$ & $2121-2133$ & $1995-2008$ & Unknown \\
\hline
\end{tabular}

${ }^{*}$ Coding sequences.

$\dagger$ Locus of fragilis glycosylation. 
(i)

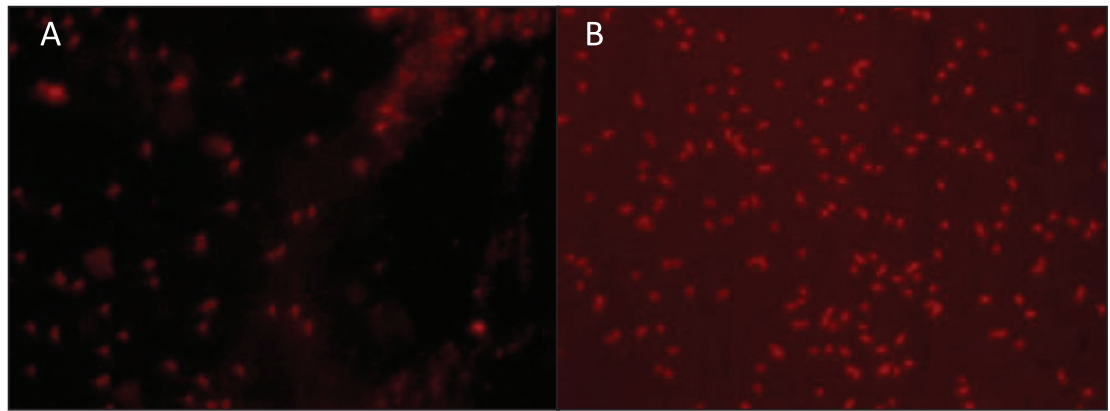

(ii)

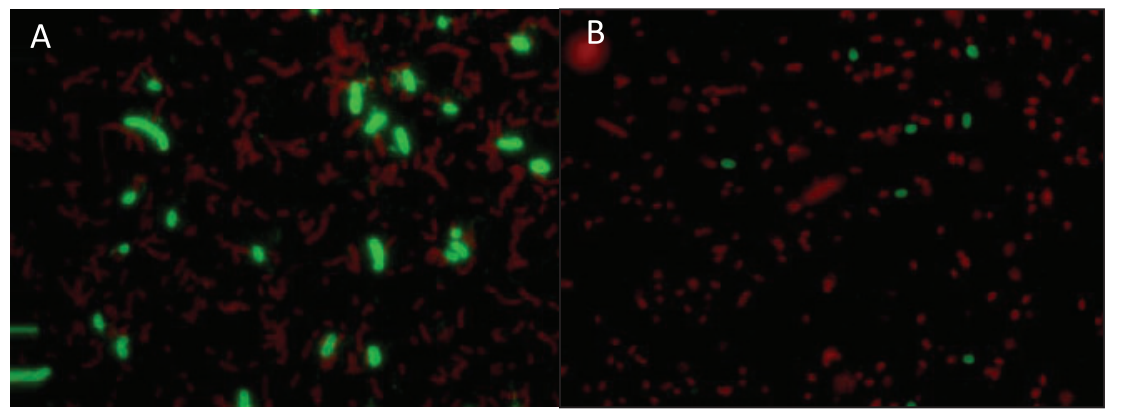

(iii)

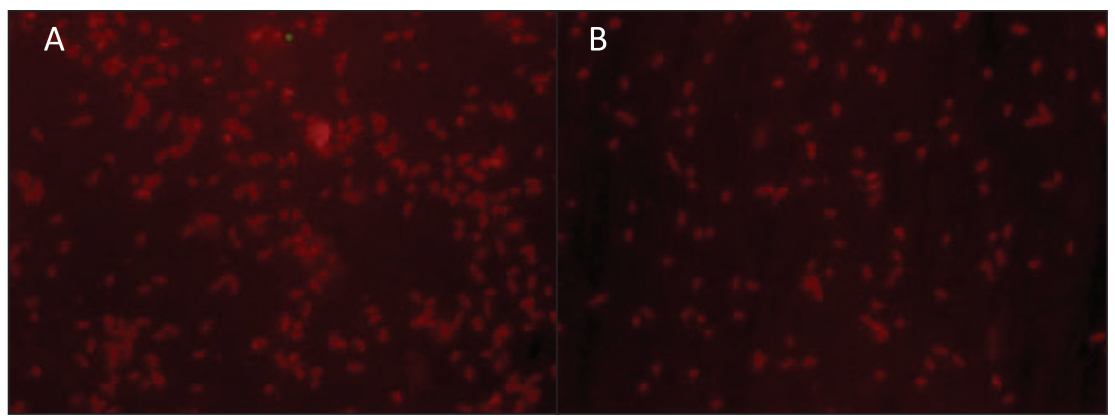

Fig. 3. Immunofluorescence microscopy of $B$. fragilis strains. (i) NCTC 9343, (ii) $638 \mathrm{R}$ and (iii) YCH46 reacted with two representative $\mathrm{mAbs},(\mathrm{A})$ and $(\mathrm{B})$, raised against $B$. fragilis $638 \mathrm{R}$, followed by anti-mouse FITC-conjugated secondary antibody, counter-stained with Evans blue. Exposure for fluorescein (green) image taken after shift of microscope slide ( $\times 100$ objective). Note the within-strain variation in epitope expression in strain 638R and the lack of cross-reactivity with NCTC 9343 and YCH46.

polymerases (Samuel \& Reeves, 2003). This suggests that these PSs can be synthesized by an E. coli group 1 or $4 \mathrm{~K}$ antigenlike mechanism (Whitfield, 2006). The 10 genes in the region downstream are conserved amongst the three strains, and include a number of putative exported and membraneassociated protein genes. This region may be relevant to the production of sugar precursors for PS synthesis. Notably, there is a conserved $B$. fragilis TAnnTTTG consensus promoter (Bayley et al., 2000) upstream of these 10 genes, indicating possible independent transcription.

PS K/11 is a novel locus, not previously reported, but present in all three sequenced strains. Again, there is considerable sequence divergence in this region (Fig. $7 \mathrm{~b}$ ). It lacks any upstream putative transcriptional regulator genes but contains a putative Wzy polymerase (BF2060 in NCTC 9343) and a putative Wzx flippase (BF2055 in NCTC 9343). This again suggests that these PSs could be synthesized by an E. coli group 1 or $4 \mathrm{~K}$ antigen-like mechanism (Whitfield, 2006).

It remains to be determined whether the PSI/9 and PSK/11 loci, which have yet to be assigned to a capsular structure, are related to expression of the LC or SC. The locus of fragilis glycosylation (lfg) region (Fletcher et al., 2009), involved in protein glycosylation, is conserved amongst the three strains (Table 1). The potential influence of other conserved genes, putatively involved with PS export but not situated within PS biosynthesis-associated loci, such as BF2000 in NCTC 9343, is unknown.

\section{PS locus comparison amongst the Bacteroidetes}

$\mathrm{Xu}$ et al. (2007) compared capsular PS loci in single genome sequences of B. vulgatus, Bacteroides thetaiotaomi- 
(i)

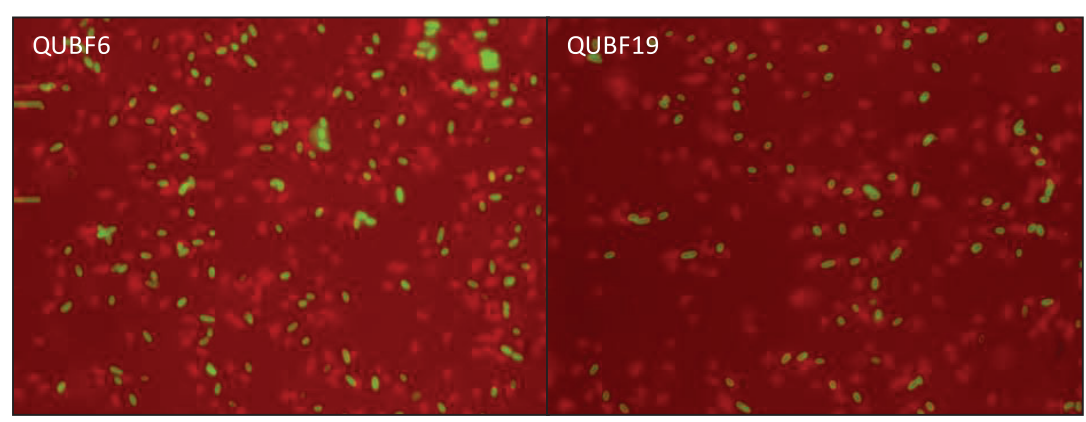

(ii)

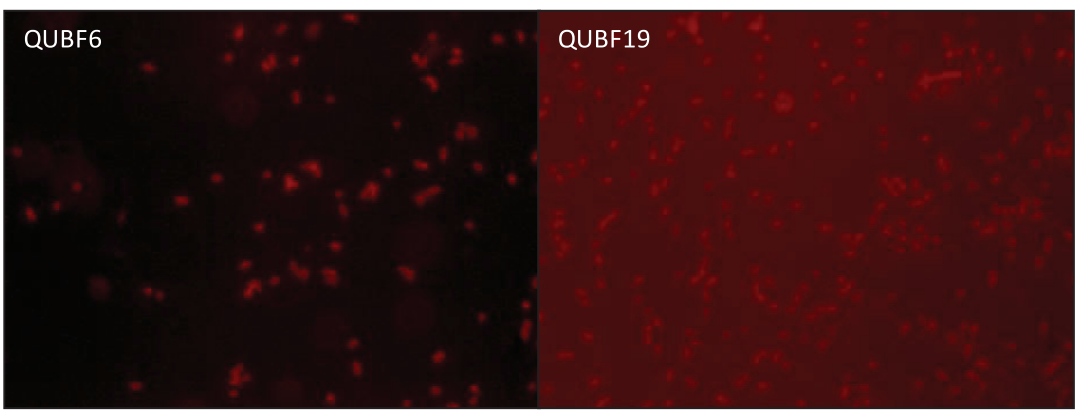

(iii)

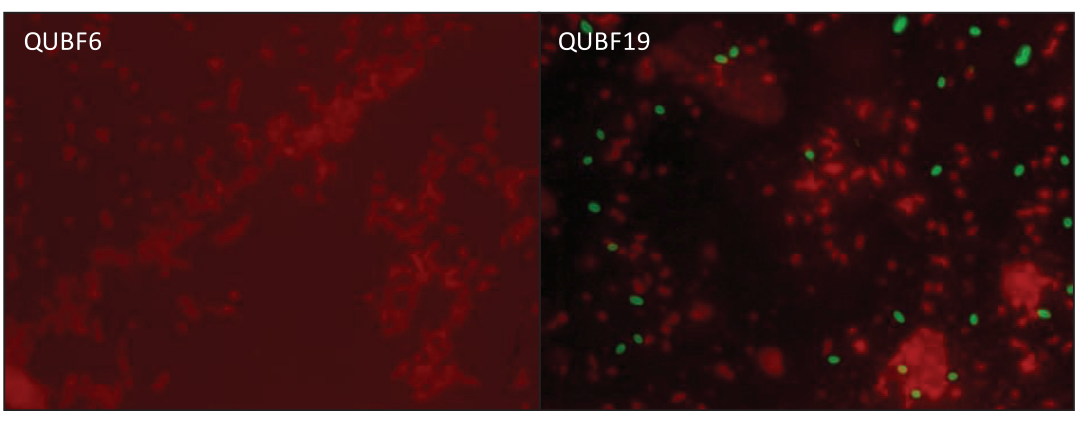

Fig. 4. Immunofluorescence microscopy of $B$. fragilis strains. (i) NCTC 9343, (ii) $638 \mathrm{R}$ and (iii) YCH46 reacted with mAbs raised against NCTC 9343 followed by anti-mouse FITC-conjugated secondary antibody, counter-stained with Evans blue. Exposure for fluorescein (green) image taken after shift of microscope slide ( $\times 100$ objective). QUBF6 reacts with PSE/1, and QUBF19 reacts with PSB/4.

cron and $P$. distasonis, which are members of the Bacteroidetes associated with the human GI tract, but are infrequently isolated from infections. They identified nine PS-associated loci in B. vulgatus, eight in B. thetaiotaomicron and 13 in $P$. distasonis. In the current study, ACT comparisons confirmed the lack of conservation with the 10 B. fragilis PS loci (results not shown). Xu et al. (2007) reported that phage insertions disrupt four $P$. distasonis capsular PS loci, including the insertion of genes between the upstream regulatory genes ( $u p c Y$ and $u p c Z$ ) and the downstream PS biosynthesis gene cluster of capsular PS locus 5 in P. distasonis. Five capsular PS biosynthesis loci of $B$. vulgatus are affected by the same phage, while two capsular loci are affected in B. thetaiotaomicron (Xu et al., 2007). The presence of a conjugative transposon inserted within one of the $B$. fragilis PS loci, YCH46 PSI/9, is another mechanism that may mediate horizontal gene transfer (Fig. 7). Conjugative transposons have been reported to be associated with the duplication and translocation of B. vulgatus PS loci (Xu et al., 2007).

\section{DNA restriction and modification ( $R-M)$ systems}

The difficulties and low efficiency of successful genetic manipulation of Bacteroides, based on the introduction of foreign DNA via electroporation, are well documented (e.g. Smith, 1995). R-M systems are proposed to protect a bacterial cell against incoming foreign DNA, particularly invading bacteriophages, but could have similar effects on DNA introduced for the purpose of genetic manipulation. The human GI tract contains a significant number and diversity of phages, as shown by metagenomic studies (Breitbart et al., 2003). Bacteriophages may have been a significant driving force in relation to the evolution of the diversity of surface 
(i) B. fragilis NCTC 9343

(a)

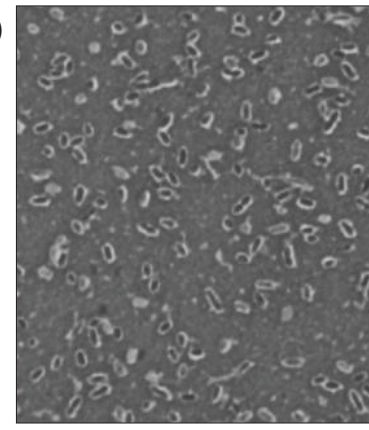

(b)

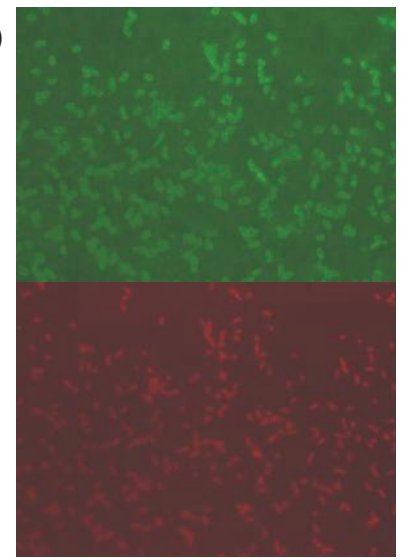

(ii) $B$. fragilis $638 \mathrm{R}$

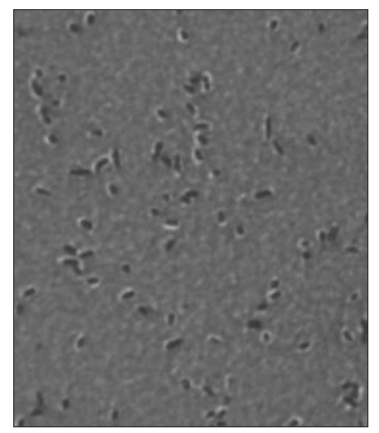

(iii) B. fragilis $\mathrm{YCH} 46$
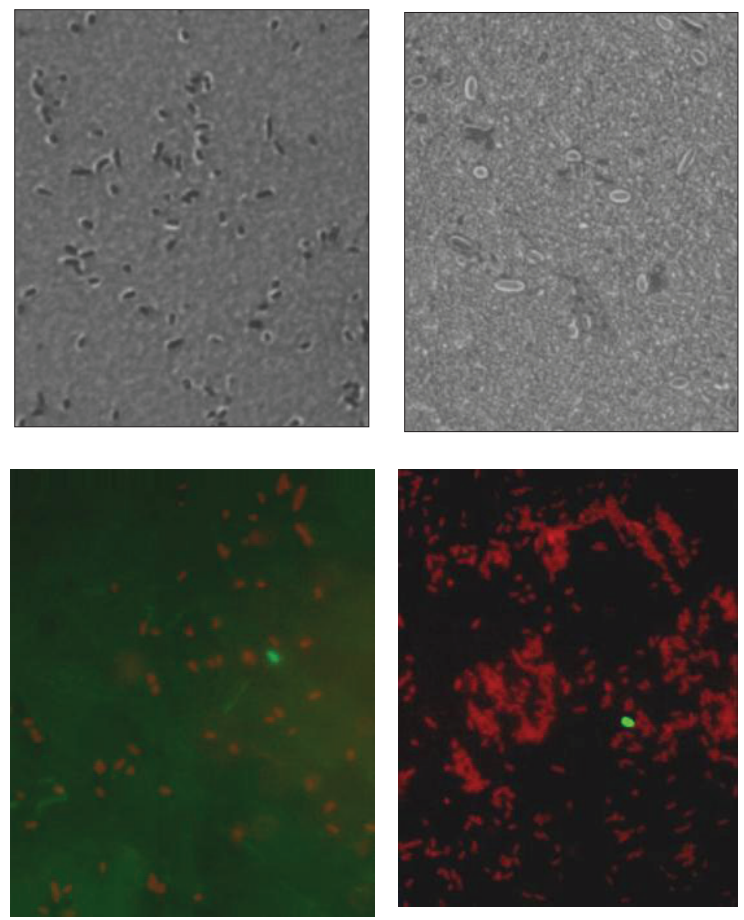

Fig. 5. Light micrographs of eosin/carbol fuchsin negative stain (a) and immunofluorescence micrographs of bacteria reacted with anti-NCTC 9343 SC mAb followed by anti-mouse FITC-conjugated secondary antibody counter-stained with Evans blue (b). (i) B. fragilis NCTC 9343, (ii) $638 \mathrm{R}$ and (iii) YCH46 sampled from the $20-40 \%$ interface of a Percoll step density gradient after centrifugation ( $\times 100$ objective). ( $b$, i) The same microscope field illustrating antibody labelling (top) and Evans blue counter stain (bottom); (b, ii) and (b, iii), combined fields. A small clear area can be observed surrounding the cells of $B$. fragilis NCTC 9343 and $\mathrm{YCH} 46$, indicating the expression of an SC. The capsule surrounding $B$. fragilis $638 \mathrm{R}$ appears more irregular. Note that $638 \mathrm{R}$ and $\mathrm{YCH} 46$ SC populations are not enriched for expression of the NCTC 9343 SC-associated epitope.

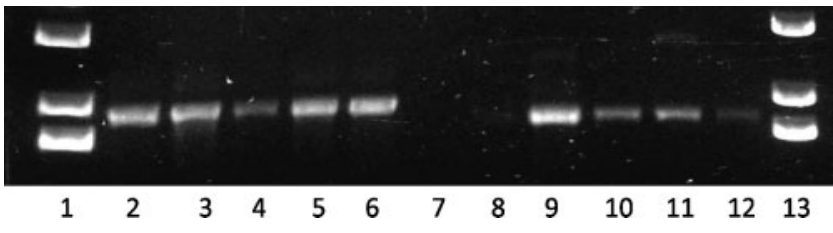

Fig. 6. PCR products obtained from genomic DNA extracted from Percoll-purified MC, SC, LC B. fragilis NCTC 9343 populations, and mixed-capsule populations from B. fragilis 638R and $\mathrm{YCH} 46$, using primer pairs designed to amplify a $1.85 \mathrm{~kb}$ product comprising the complete BF2782 gene with its promoter in the 'ON' orientation (lanes 2-6) and a $1.75 \mathrm{~kb}$ product comprising the complete BF2782 gene with its promoter in the 'OFF' orientation (lanes 8-12). Lanes: 2 and 8, NCTC 9343 LC PCR; 3 and 9, NCTC 9343 SC PCR; 4 and 10, NCTC 9343 MC PCR; 5 and 11, 638R PCR; 6 and 12, YCH46 PCR; 1 and 13, 1 kb Plus DNA ladder (Invitrogen). Lane 7 is empty. Note the lack of product with the OFF orientation primers in the NCTC 9343 LC population, lane 8. phenotypes described above. The genome of $B$. fragilis NCTC 9343 specifies three type I and two type III R-M systems, as compared with the 638R genome, which specifies two type I, one type IIS and one type III R-M systems.

Type I R-M systems consist of three subunits, HsdR, HsdM and HsdS, the last of which dictates the DNA-binding specificity of the methyltransferase and the endonuclease. The NCTC 9343 genome contains a locus (BF1839-1842) which shows phase-variable expression of an HsdS subunit with eight different possible polypeptide combinations, which in turn provides eight different DNA-recognition specificities (Shufflon BB). A related hsdS shufflon (BF 638R 1146-1149) is present in the genome of 638R, but is located in a different chromosomal location, and the encoded polypeptides share between 27 and $49 \%$ identity with the products of the NCTC 9343 shufflon. This indicates that the shufflons have a common origin, although the coding sequences have subsequently diverged.

In NCTC 9343, the shufflon contains four sets of inverted repeats that are associated with site-specific rearrangement 
(a)

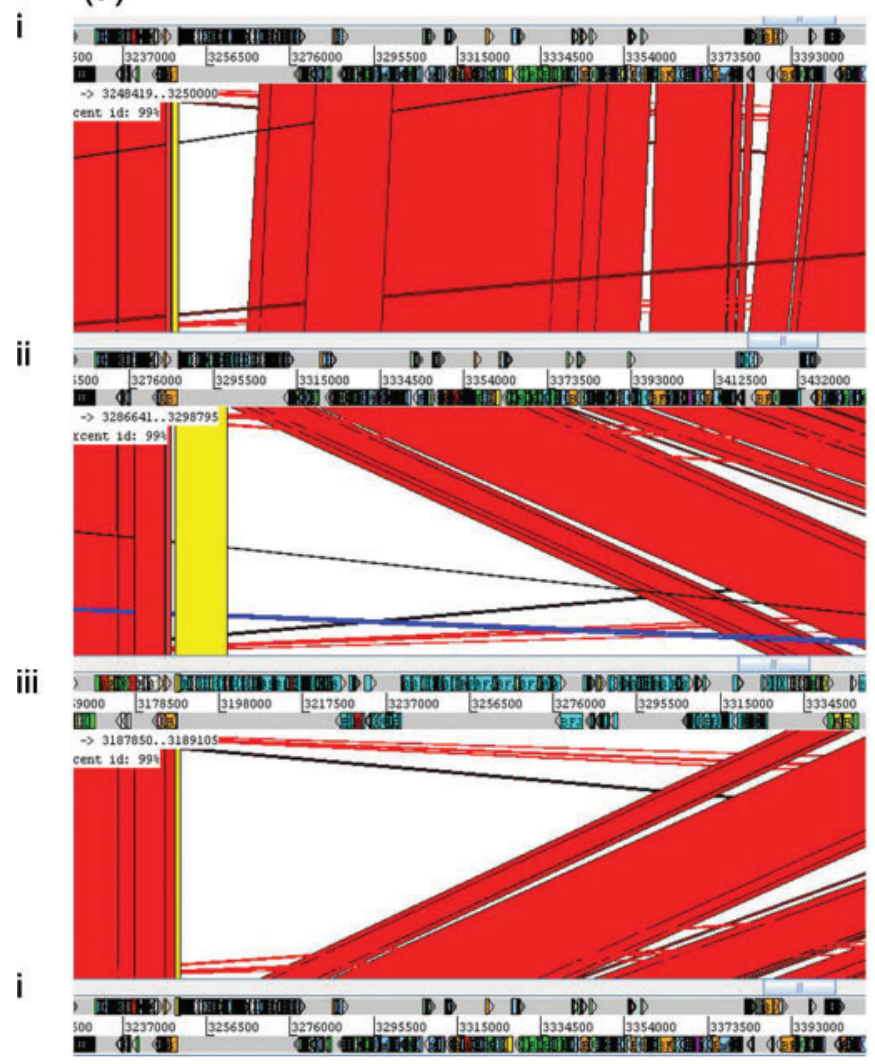

(b)

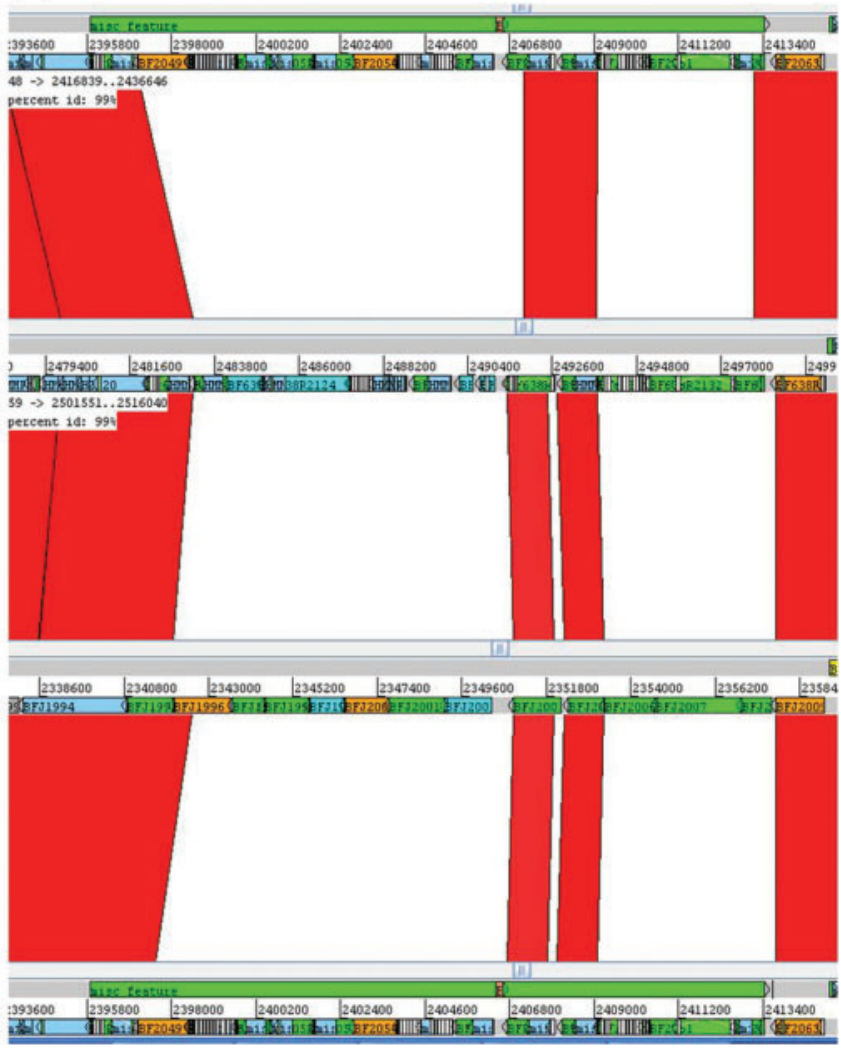

Fig. 7. Four-way ACT genome comparison of PS-associated loci. (a) PSI/9: black arrows, inserted conjugative transposonassociated regions in YCH46; (b) PSK/11 of B. fragilis. (i) NCTC 9343, (ii) 638R, (iii) YCH46. Red or yellow coloration between sequences indicates sequence identity, no colour indicates divergent sequence.

of the target recognition domains (TRDs) within the encoded HsdS proteins. One of these repeats (5-'CTTATTGATGAACGTATCGCTACCCAAAACAAAATCAT-3') is conserved (32/38 bp) within the 638R shufflon, suggesting that a common recombinase is responsible for inversion for at least two of the TRDs. In the shufflons of both genomes, genes encoding tyrosine integrases with $61 \%$ identity are present immediately adjacent to the last $h s d S$ gene, reinforcing the notion that they mediate this subset of inversions. Both type I R-M systems in 638R are contained within a putative $50 \mathrm{~kb}$ horizontally acquired island, ICE2, which is possibly a prophage relic. The type IIS R-M bifunctional enzyme is encoded within a chromosomal island in the analogous position to the island that encodes the type I shufflon in NCTC 9343. The type III-specifying locus in 638R (BF 638R 1130 and 1131) is the only common R-M system amongst the three sequenced strains of $B$. fragilis. Together, these observations suggest that the common genome backbone of $B$. fragilis specifies a type III $\mathrm{R}-\mathrm{M}$ system and that diversification of $\mathrm{R}-\mathrm{M}$ specificities within strains is achieved by horizontal gene transfer.

The extent and variety of R-M systems in $638 \mathrm{R}$ will act to prevent phage infection but will also be a barrier to horizontal gene transfer. At least two of the conjugative transposons that reside in the genome have evaded this barrier by encoding homologues of the ArdA antirestriction protein that mimics B-form DNA and blocks the binding site of type I R-M systems (McMahon et al., 2009).

In addition to the classical R-M systems, the NCTC 9343 genome encodes proteins with homology to the type IV restriction endonuclease $\mathrm{McrBC}$, which cleaves specific DNA sequences modified with 5-methylcytosine. These genes are not present in the genome of 638R, and their absence, despite the potential expression of nine different type I R-M specificities, might contribute to the successful transformation of the strain using DNA derived from $E$. coli K-12 that is usually modified by the Dcm methyltransferase.

The R-M systems encoded within B. fragilis are undoubtedly responsible for the low efficiency of successful genetic manipulation based on the introduction of foreign DNA via electroporation, in particular in relation to NCTC 9343 (e.g. Smith, 1995). This is also in keeping with the observed improvement of electroporation efficiency when the bacteriophage T7 Ocr (overcomes classical restriction) 
protein, a DNA mimic, is included in the electroporation mix (Patrick et al., 2009). Ocr protein reduces the activity of type I R-M systems in a range of bacteria and archaea (Dryden, 2006).

\section{General genomic comparisons}

The general characteristics of the three genomes are broadly similar (Table 2). Regions of low or no sequence identity amongst the three individual genomes account for approximately $11 \%$ of the genome sequences. These nonidentical regions include the PS regions as discussed above, and differences in inserted extra-chromosomal elements (ICEs) such as conjugative transposons and prophages. Differences in numbers of ICEs also largely account for differences in chromosomal size between the strains.

Invertible regions. A major unusual feature of the $B$. fragilis genome, first identified in NCTC 9343, is the diversity and extent of invertible DNA elements within the genome. These include 16 fragilis invertible (fin) promoter regions, in addition to those controlling PS loci and three complex invertible DNA shufflons (Cerdeño-Tárraga et al., 2005). Conserved fin regions, not associated with PS loci, are evident throughout all three genomes. Invertible regions $\mathrm{R}$ and $\mathrm{S}$, which involve putative outer-membrane proteins, are present in all three strains. An overlap of inverted repeat regions in $\mathrm{R}$ and $\mathrm{S}$ is evident in the comparison of the three genomes, suggesting that these two regions together may generate multiple rearrangements, thus forming a further complex gene shufflon (Fig. 8). The invertible region $\mathrm{Q}$, which causes alteration of the orientation of two hypothetical proteins with or against the direction of transcription of the surrounding genes, is, however, missing from 638R, as is the complete putative phage-associated region. The previously described complex invertible region shufflons CC and EE in NCTC 9343 (Cerdeño-Tárraga et al., 2005) are present in both 638R and YCH46, although only in NCTC 9343 does the shufflon EE contain an inserted conjugative transposon. The function of these many potentially variable surface

Table 2. General genomic comparison of $B$. fragilis NCTC 9343, 638R and $\mathrm{YCH} 46$

\begin{tabular}{|lccc|}
\hline Parameter & \multicolumn{3}{c|}{ Strain } \\
\cline { 2 - 4 } & NCTC 9343* & $\mathbf{6 3 8 R}$ & YCH46 $\dagger$ \\
\hline Genome size (bp) & 5205140 & 5373121 & 5277274 \\
CDSs $\ddagger$ & 4241 & 4308 & 4578 \\
Plasmid & pBF9343 & 0 & pBFY46 \\
& $(36.6 \mathrm{~kb})$ & & $(33.7 \mathrm{~kb})$ \\
\hline
\end{tabular}

*Cerdeño-Tárraga et al. (2005).

$\dagger$ Kuwahara et al. (2004).

¥Coding sequences. proteins is unknown, but it would be interesting to determine whether they are linked to bacteriophage adsorption.

Origin of $638 \mathrm{R}$ rifampicin resistance. The spontaneous rifampicin-resistant mutant strain 638R (Privitera et al., 1979) was generated at the Institut Pasteur from a clinical isolate originating from Chicago (Stiffler et al., 1974). Rifampicin binds within the DNA/RNA channel of the $\beta$ subunit in RNA polymerase and blocks the exit path for the nascent transcript. There is a single putative $r p o C$ RNA polymerase $\beta$ subunit homologue in all three $B$. fragilis genomes. Comparison of the nucleotide sequence reveals 12 single base pair changes; however, only one results in an altered amino acid. A transition at base pair 1475 results in the loss of a conserved serine, which is replaced with a phenylalanine. Serine 492 in Bf-RpoC corresponds to serine 411 in the Thermus aquaticus (Taq) RNA polymerase, which forms a hydrogen bond with oxygen 2 in the naphthol ring of rifampicin (Campbell et al., 2001). Mutations that change serine 411 to phenylalanine in Taq RNA polymerase make the enzyme insensitive to rifampicin; this is also true for the corresponding S531F change in the $\beta$ subunit of $E$. coli RNA polymerase (Campbell et al., 2005).

\section{Conclusion}

Each B. fragilis strain examined has 10 separate divergent PS regions, each potentially capable of generating an extracellular PS, of which only two are conserved between two of the strains. There is, therefore, the potential for the expression of 28 different PSs amongst these three strains. This represents an unprecedented diversity of PSs within such a limited number of strains. For example, in Streptococcus pneumoniae, although there are 90 different PS loci, each confers a single serotype on a single strain (Bentley et al., 2006).

Surface component variation may enhance survival when $B$. fragilis escapes into other body sites and generates opportunistic infection, but this does not fully explain why $B$. fragilis is more commonly associated with opportunistic infections, including bacteraemia, when compared with other members of the Bacteroidetes. Undoubtedly a number of factors in combination will contribute to virulence. For example, a fibrinogen-binding protein identified in NCTC 9343 (Houston et al., 2010) is conserved amongst the three sequenced $B$. fragilis strains NCTC 9343, 638R and YCH46, which were isolated from abscess and bacteraemia patients, but is not conserved in $B$. vulgatus and shares only $33 \%$ identity to a putative cell surface antigen of B. thetaiotaomicron (BT_1896), both of which are rarely isolated from clinical infections.

The multiple and diverse PS biosynthesis loci in B. fragilis and other members of the Bacteroidetes indicate a strong selective pressure in favour of PS variation within the human GI tract. It is likely that these have arisen from a 


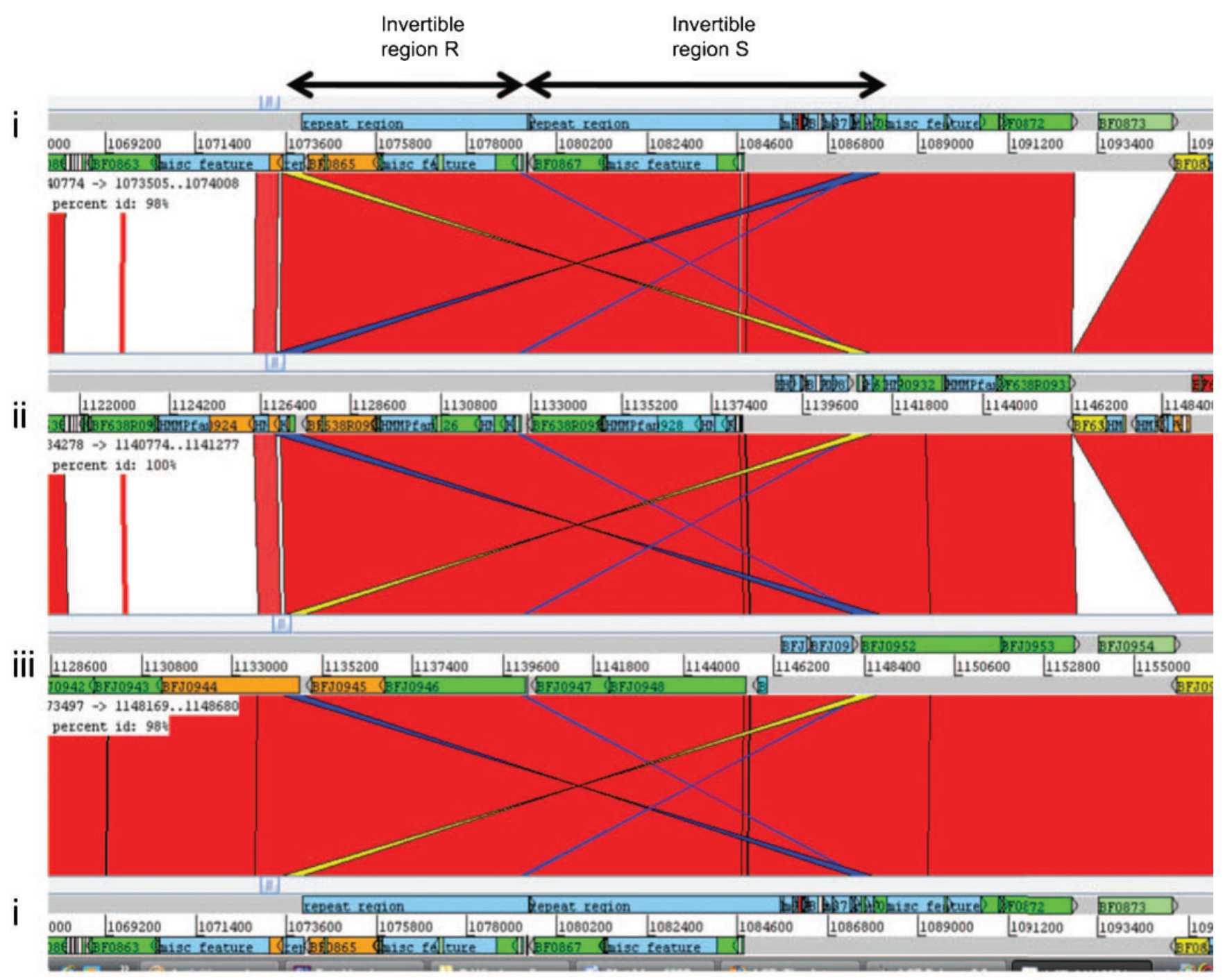

Fig. 8. Comparison of invertible regions $\mathrm{R}$ and $\mathrm{S}$ (arrows), active in the NCTC 9343 shotgun sequence of $B$. fragilis. (i) NCTC 9343, (ii) 638R, (iii) $\mathrm{YCH} 46$. Red coloration between sequences indicates sequence identity, no colour indicates divergent sequence, dark blue/yellow indicates inverted sequences. Note the sequence identity of inverted sequences in $\mathrm{R}$ and $\mathrm{S}$, which suggests that these two regions form a complex intergenic shufflon.

divergence of operons over evolutionary time combined with more rapid changes arising from horizontal transmission amongst cells representing a pan-genome with an extensive pool of different PS loci. Interestingly, horizontal gene transfer has occurred despite the presence of a significant barrier in the form of diverse and phase-variable DNA R-M systems.

Given the number of phage-related regions within the $B$. fragilis genome, and the presence of diverse PS loci in Bacteroidetes infrequently associated with opportunistic infection, such as B. vulgatus and P. distasonis, it may be that a major driver of amongst-strain PS locus diversity, within-strain surface protein and PS diversity generated by DNA inversion (Cerdeño-Tárraga et al., 2005), and extensive DNA restriction and modification, is the prevention of bacteriophage adsorption and infection within the GI tract.

\section{ACKNOWLEDGEMENTS}

This work was supported by the Wellcome Trust (grant no. 061696) through its Beowulf Genomics initiative. We would like to acknowledge the support of the Wellcome Trust Sanger Institute core sequencing and informatics groups. S.P. thanks M. Larkin (QUESTOR, Queen's University of Belfast) for the use of his laboratory and advice on DNA extraction methods. We are grateful to C. J. Smith, East Carolina University, USA, for supplying strain 638R, and T. Kuwahara, University of Tokushima, Japan, for supplying B. fragilis YCH46. S. H. and J.M. were in receipt of Department of Employment and Learning Northern Ireland Studentships. 


\section{REFERENCES}

Ausubel, F. M., Brent, R., Kingston, R. E., Moore, D. D., Seidman, J. G., Smith, J. A. \& Struhl, K. (editors) (1992). Current Protocols in Molecular Biology. New York: Wiley.

Bayley, D. P., Rocha, E. R. \& Smith, C. J. (2000). Analysis of cepA and other Bacteroides fragilis genes reveals a unique promoter structure. FEMS Microbiol Lett 193, 149-154.

Bentley, S. D., Aanensen, D. M., Mavroidi, A., Saunders, D., Rabbinowitsch, E., Collins, M., Donohoe, K., Harris, D., Murphy, L. \& other authors (2006). Genetic analysis of the capsular biosynthetic locus from all 90 pneumococcal serotypes. PLoS Genet 2, e31.

Breitbart, M., Hewson, I., Felts, B., Mahaffy, J. M., Nulton, J., Salamon, P. \& Rohwer, F. (2003). Metagenomic analyses of an uncultured viral community from human feces. J Bacteriol 185, 62206223.

Campbell, E. A., Korzheva, N., Mustaev, A., Murakami, K., Nair, S., Goldfarb, A. \& Darst, S. A. (2001). Structural mechanism for rifampicin inhibition of bacterial RNA polymerase. Cell 104, 901912.

Campbell, E. A., Pavlova, O., Zenkin, N., Leon, F., Irschik, H., Jansen, R., Severinov, K. \& Darst, S. A. (2005). Structural, functional, and genetic analysis of sorangicin inhibition of bacterial RNA polymerase. EMBO J 24, 674-682.

Carver, T. J., Rutherford, K. M., Berriman, M., Rajandream, M. A., Barrell, B. G. \& Parkhill, J. (2005). ACT: the Artemis comparison tool. Bioinformatics 21, 3422-3423.

Cerdeño-Tárraga, A. M., Patrick, S., Crossman, L. C., Blakely, G., Abratt, V., Lennard, N., Poxton, I., Duerden, B., Harris, B. \& other authors (2005). Extensive DNA inversions in the $B$. fragilis genome control variable gene expression. Science 307, 1463-1465.

Chatzidaki-Livanis, M., Coyne, M. J., Roche-Hakansson, H. \& Comstock, L. E. (2008). Expression of a uniquely regulated extracellular polysaccharide confers a large-capsule phenotype to Bacteroides fragilis. J Bacteriol 190, 1020-1026.

Cheng, C. W., Lin, H. S., Ye, J. J., Yang, C. C., Chiang, P. C., Wu, T. S. \& Lee, M. H. (2009). Clinical significance of and outcomes for Bacteroides fragilis bacteremia. J Microbiol Immunol Infect 42, 243 250.

Coyne, M. J., Tzianabos, A. O., Mallory, B. C., Carey, V. J., Kasper, D. L. \& Comstock, L. E. (2001). Polysaccharide biosynthesis locus required for virulence of Bacteroides fragilis. Infect Immun 69, 43424350 .

Drummelsmith, J. \& Whitfield, C. (2000). Translocation of group 1 capsular polysaccharide to the surface of Escherichia coli requires a multimeric complex in the outer membrane. EMBO J 19, 5766.

Dryden, D. T. (2006). DNA mimicry by proteins and the control of enzymatic activity on DNA. Trends Biotechnol 24, 378-382.

Fletcher, C. M., Coyne, M. J., Villa, O. F., Chatzidaki-Livanis, M. \& Comstock, L. E. (2009). A general O-glycosylation system important to the physiology of a major human instestinal symbiont. Cell 137, 321-331.

Houston, S., Blakely, G. W., McDowell, A., Martin, L. \& Patrick, S. (2010). Binding and degradation of fibrinogen by Bacteroides fragilis and characterization of a $54 \mathrm{kDa}$ fibrinogen-binding protein. Microbiology 156, 2516-2526.

Katsandri, A., Papaparaskevas, J., Pantazatou, A., Petrikkos, G. L., Thomopoulos, G., Houhoula, D. P. \& Avlamis, A. (2006). Two cases of infections due to multidrug-resistant Bacteroides fragilis group strains. J Clin Microbiol 44, 3465-3467.
Kuwahara, T., Yamashita, A., Hirakawa, H., Nakayama, H., Toh, H., Okada, N., Kuhara, S., Hattori, M., Hayashi, T. \& Ohnishi, Y. (2004). Genomic analysis of Bacteroides fragilis reveals extensive DNA inversions regulating cell surface adaptation. Proc Natl Acad Sci U S A 101, 14919-14924.

Lutton, D. A., Patrick, S., Crockard, A. D., Stewart, L. D., Larkin, M. J., Dermott, E. \& McNeill, T. A. (1991). Flow cytometric analysis of withinstrain variation in polysaccharide expression by Bacteroides fragilis by use of murine monoclonal antibodies. J Med Microbiol 35, 229-237.

Mazmanian, S. K., Round, J. L. \& Kasper, D. L. (2008). A microbial symbiosis factor prevents intestinal inflammatory disease. Nature $\mathbf{4 5 3}$, 620-625.

McMahon, S. A., Roberts, G. A., Johnson, K. A., Cooper, L. P., Liu, H., White, J. H., Carter, L. G., Sanghvi, B., Oke, M. \& other authors (2009). Extensive DNA mimicry by the ArdA anti-restriction protein and its role in the spread of antibiotic resistance. Nucleic Acids Res 37, 48874897.

Patrick, S. (2002). Bacteroides. In Molecular Medical Microbiology, pp. 1921-1948. Edited by M. Sussman. London: Academic Press.

Patrick, S. \& Duerden, B. I. (2006). Gram-negative non-spore forming obligate anaerobes. In Principles and Practice of Clinical Bacteriology, 2nd edn, pp. 541-556. Edited by S. H. Gillespie \& P. Hawkey. London: Wiley.

Patrick, S. \& Reid, J. H. (1983). Separation of capsulate and noncapsulate Bacteroides fragilis on a discontinuous density gradient. J Med Microbiol 16, 239-241.

Patrick, S., Reid, J. H. \& Coffey, A. (1986). Capsulation of in vitro and in vivo grown Bacteroides species. J Gen Microbiol 132, 1099-1109.

Patrick, S., Stewart, L. D., Damani, N., Wilson, K. G., Lutton, D. A., Larkin, M. J., Poxton, I. \& Brown, R. (1995). Immunological detection of Bacteroides fragilis in clinical samples. J Med Microbiol 43, 99109.

Patrick, S., Gilpin, D. \& Stevenson, L. (1999). Detection of intrastrain antigenic variation of Bacteroides fragilis surface polysaccharides by monoclonal antibody labelling. Infect Immun 67, 4346-4351.

Patrick, S., Parkhill, J., McCoy, L. J., Lennard, N., Larkin, M. J., Collins, M., Sczaniecka, M. \& Blakely, G. (2003). Multiple inverted DNA repeats of Bacteroides fragilis that control polysaccharide antigenic variation are similar to the hin region inverted repeats of Salmonella typhimurium. Microbiology 149, 915-924.

Patrick, S., Houston, S., Thacker, Z. \& Blakely, G. W. (2009). Mutational analysis of genes implicated in LPS and capsular polysaccharide biosynthesis in the opportunistic pathogen Bacteroides fragilis. Microbiology 155, 1039-1049.

Privitera, G., Dublanchet, A. \& Sebald, M. (1979). Transfer of multiple antibiotic resistance between subspecies of Bacteroides fragilis. J Infect Dis 139, 97-101.

Qin, J., Li, R., Raes, J., Arumugam, M., Burgdorf, K. S., Manichanh, C., Nielsen, T., Pons, N., Levenez, F. \& other authors (2010). A human gut microbial gene catalogue established by metagenomic sequencing. Nature 464, 59-65.

Reid, J. H. \& Patrick, S. (1984). Phagocytic and serum killing of capsulate and non-capsulate Bacteroides fragilis. J Med Microbiol 17, 247-257.

Reid, J. H., Patrick, S. \& Tabaqchali, S. (1987). Immunochemical characterization of a polysaccharide antigen of Bacteroides fragilis with an IgM monoclonal antibody. J Gen Microbiol 133, 171-179.

Sakamoto, M. \& Benno, Y. (2006). Reclassification of Bacteroides distasonis, Bacteroides goldsteinii and Bacteroides merdae as Parabacteroides distasonis gen. nov., comb. nov., Parabacteroides goldsteinii comb. nov. and Parabacteroides merdae comb. nov. Int J Syst Evol Microbiol 56, 1599-1605. 
Samuel, G. \& Reeves, P. (2003). Biosynthesis of O-antigens: genes and pathways involved in nucleotide sugar precursor synthesis and $\mathrm{O}$ antigen assembly. Carbohydr Res 338, 2503-2519.

Smith, C. J. (1995). Genetic transformation of Bacteroides fragilis spp. using electroporation. In Methods in Molelcular Biology: Electroporation Protocols for Microorganisms, pp. 161-169. Edited by J. A. Nickloff. Totowa, NJ: Humana Press.

Stiffler, P. W., Keller, R. \& Traub, N. (1974). Isolation and characterization of several cryptic plasmids from clinical isolates of Bacteroides fragilis. J Infect Dis 130, 544-548.

Tally, F. P., Snydman, D. R., Shimell, M. J. \& Malamy, M. H. (1982). Characterization of pBFTM10, a clindamycin-erythromycin resistance transfer factor from Bacteroides fragilis. J Bacteriol 151, 686-691.

van der Woude, M. W. \& Baumler, A. J. (2004). Phase and antigenic variation in bacteria. Clin Microbiol Rev 17, 581-611.

Van Tassell, R. L. \& Wilkins, T. D. (1978). Isolation of auxotrophs of Bacteroides fragilis. Can J Microbiol 24, 1619-1621.
Vimr, E. R. \& Steenbergen, S. M. (2006). Mobile contingency locus controlling Escherichia coli K1 polysialic acid capsule acetylation. Mol Microbiol 60, 828-837.

Wareham, D. W., Wilks, M., Ahmed, D., Brazier, J. S. \& Millar, M. (2005). Anaerobic sepsis due to multidrug-resistant Bacteroides fragilis: microbiological cure and clinical response with linezolid therapy. Clin Infect Dis 40, e67-e68.

Wexler, H. M. (2007). Bacteroides: the good, the bad, and the nittygritty. Clin Microbiol Rev 20, 593-621.

Whitfield, C. (2006). Biosynthesis and assembly of capsular polysaccharides in Escherichia coli. Annu Rev Biochem 75, 39-68.

Xu, J., Mahowald, M. A., Ley, R. E., Lozupone, C. A., Hamady, M., Martens, E. C., Henrissat, B., Coutinho, P. M., Minx, P. \& other authors (2007). Evolution of symbiotic bacteria in the distal human intestine. PLoS Biol 5, e156.

Edited by: P. W. O'Toole 